\title{
Soft and Stretchable Liquid Metal Composites with Shape Memory and Healable Conductivity
}

Priyanuj Bhuyan ${ }^{+1,2}$, Yuwen Weit1,2, Dongho Sin ${ }^{1,2}$, Jaesang Yư ${ }^{3}$, Changwoon Nah ${ }^{1,4}$, Kwang-Un Jeong ${ }^{1,2}$, Michael. D. Dickey ${ }^{5}$, Sungjune Park ${ }^{1,2 *}$

${ }^{1}$ Department of Polymer-Nano Science and Technology, Jeonbuk National University, Jeonju 54896, Korea

2Department of Nano Convergence Engineering, Jeonbuk National University, Jeonju 54896, Korea

${ }^{3}$ Institute of Advanced Composite Materials, Korea Institute of Science and Technology (KIST), 92 Chudong-ro, Bongdong-eup, Wanju-gun, Jeonbuk 55324, Korea

${ }^{4}$ Department of Bio-Nanotechnology and Bio-Convergence Engineering, Jeonbuk National University, Jeonju 54896, Korea

${ }^{5}$ Department of Chemical and Biomolecular Engineering, North Carolina State University,

911 Partners Way, Raleigh, NC 27695, USA

*Corresponding Author email : s.park@jbnu.ac.kr 


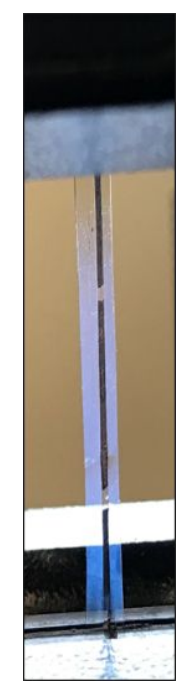

Figure S1. Image showing serial fracture of the solid wire of the film made of Sylgar 184 upon stretching.

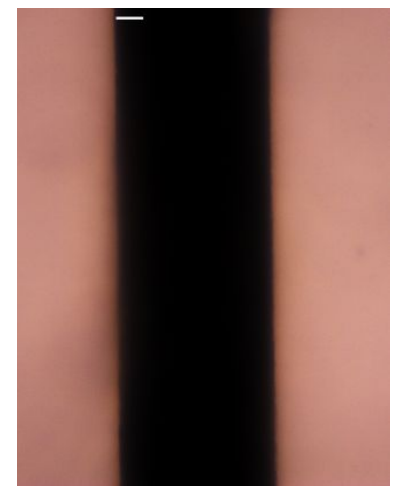

Figure S2. Microscopic image of gallium-filled microchannel of the Ecoflex film (Figure 2b). Average microscopic width of the microchannel is $249.8 \mu \mathrm{m}$. Average values of the width of microchannels were calculated by taking 10 random width values along the length of the channel. Scale bar, $40 \mu \mathrm{m}$. 


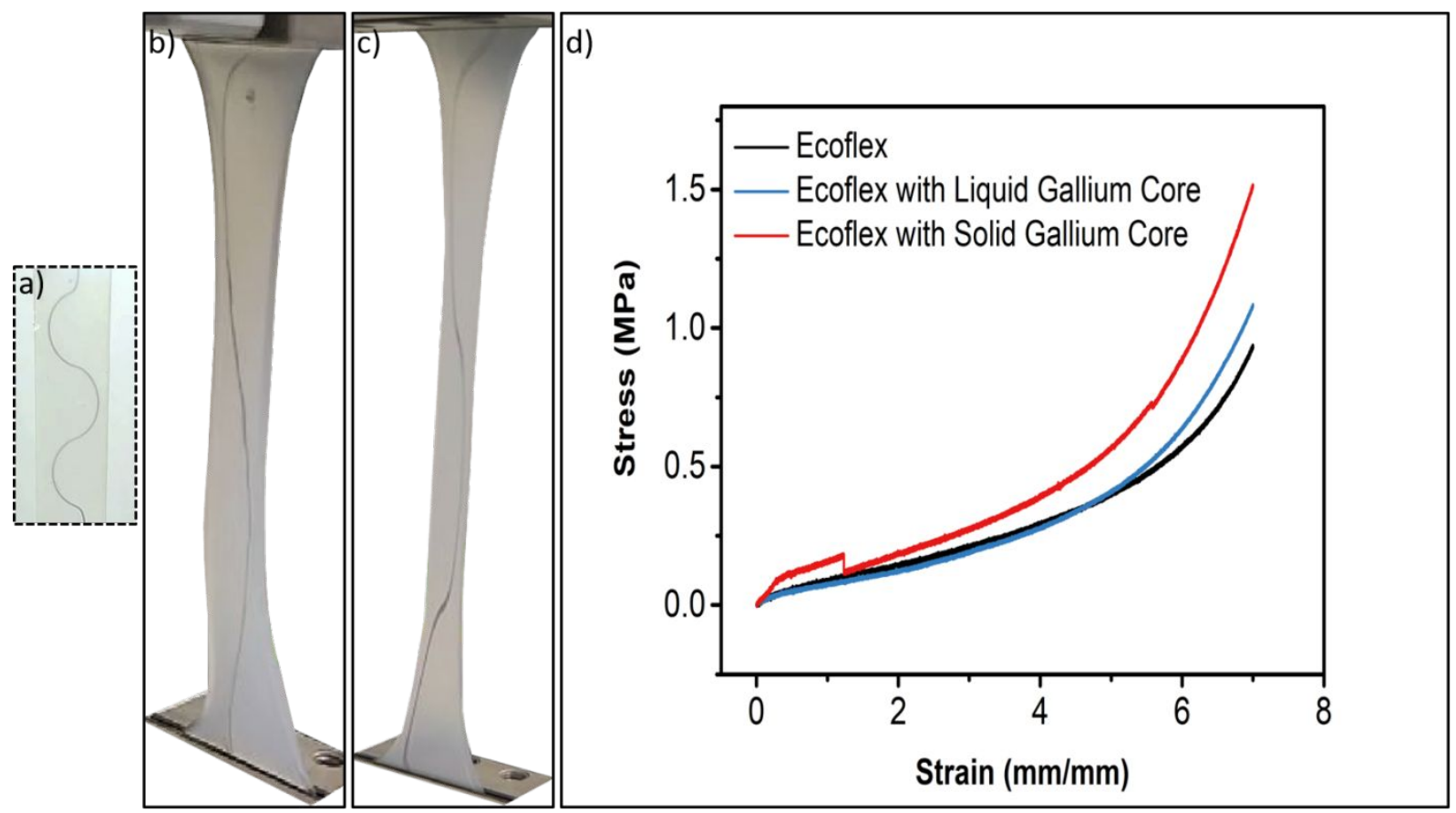

Figure S3. (a-c) Ecoflex films with sinusoidal shaped microchannels having a diameter of $250 \mu \mathrm{m}$ filled with liquid gallium. (a) Ecoflex film with entrapped sinusoidal liquid gallium wire. (b) Ecoflex film with solid metal wire upon stretching. (c) Ecoflex film with liquid metal wire showing localized narrowing upon stretching. d) Stress-strain plots of polymer films compared to that of Ecoflex film with the same dimensions. 

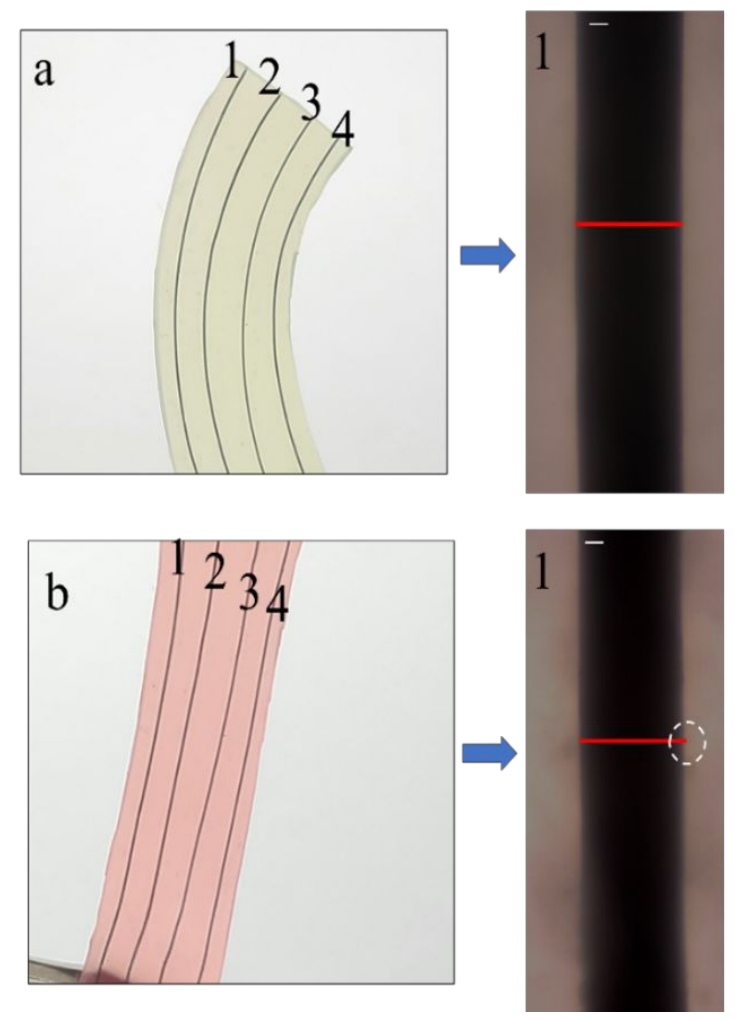
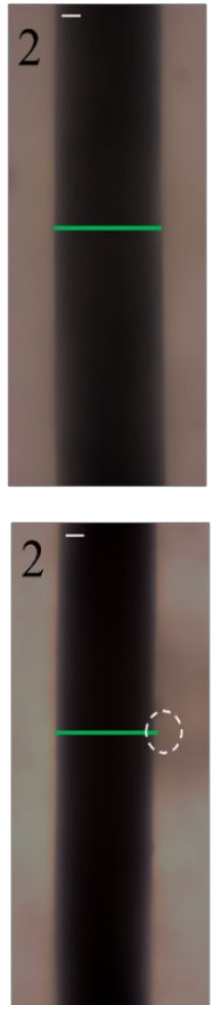
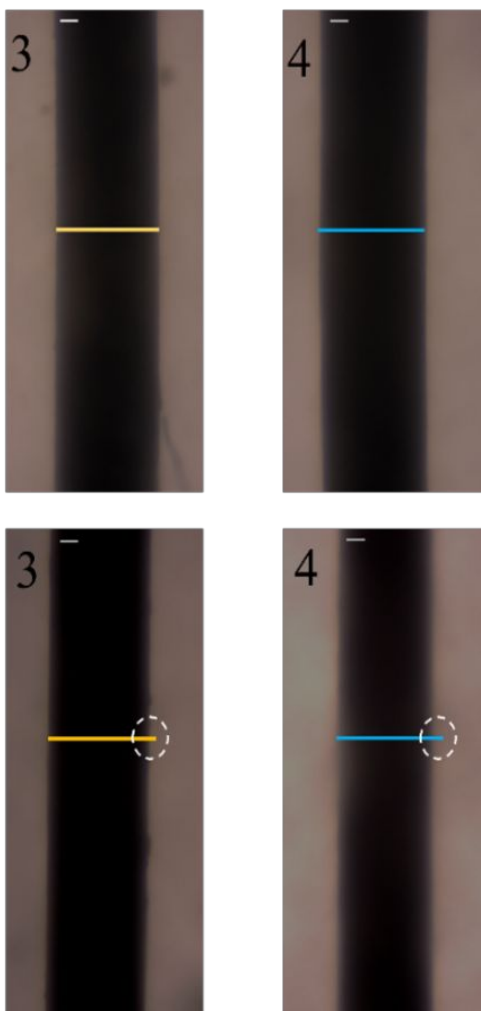

Figure S4. (a) Ecoflex film with liquid gallium wire filled into microchannels in a slack state. Corresponding numbers refer to the microscopic image of each channel from high radius end to the low radius end of the film (Widths: a1, $249.8 \mu \mathrm{m}$; a2, 249.6 $\mu \mathrm{m}$; a3, 249.8 $\mu \mathrm{m}$; a4, $249.7 \mu \mathrm{m}$ ). (b) Ecoflex film after stretching and freezing gallium cores. Corresponding numbers refer to the microscopic image of each channel from a high radius end to a low radius end of the film (Widths: b1, $247.3 \mu \mathrm{m}$; b2, $242.7 \mu \mathrm{m}$; b3, $230.6 \mu \mathrm{m}$; b4, $221.7 \mu \mathrm{m})$. To distinguish between widths of microchannels before and after programming, widths are marked with same colors and the increase in widths are marked with white circles. Average values of widths of microchannels were calculated by taking 10 random width values along the length of the channel. Scale bar, $40 \mu \mathrm{m}$. 

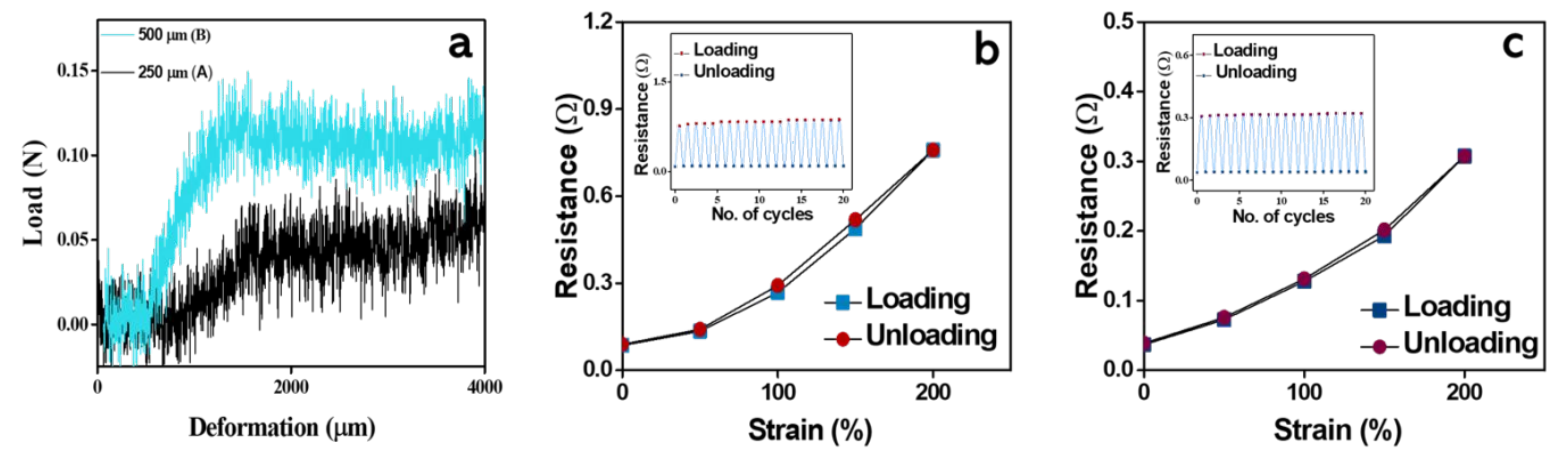

Figure S5. (a)Three point bending test result of the elastomeric polymer films A and B (microchannel with diameter of 250 and $500 \mu \mathrm{m}$, respectively) with solidified gallium wires. The loading force of the film B is higher than that of the film A due to the presence of solidified gallium wires with larger diameter. When the gallium wires are in liquid state, the films are slack, due to which the bending test cannot be performed. (b-c) Resistance vs strain of the films (b) A and (c) B ( Inset figures show resistance change for 20 cycles of loading-unloading). 


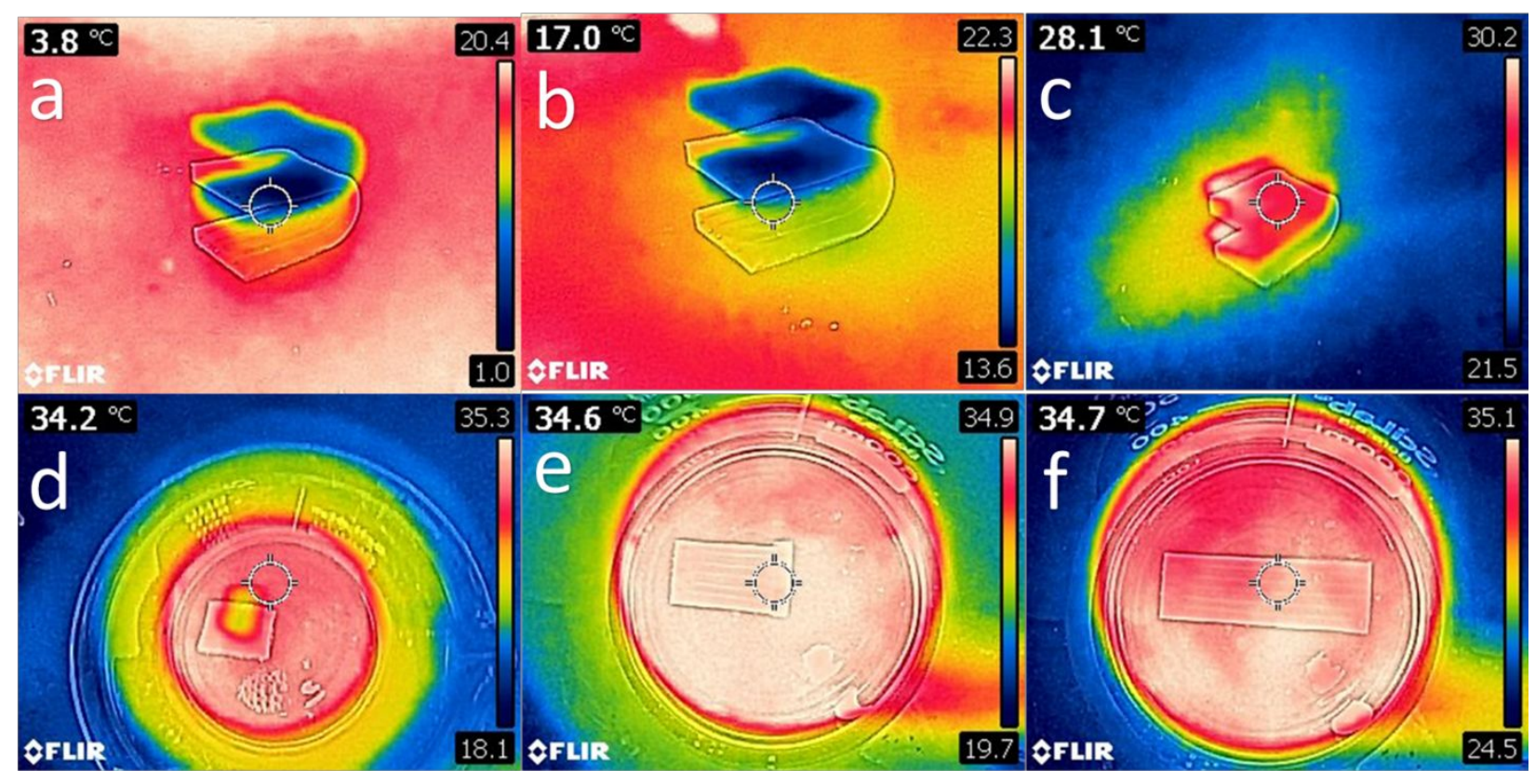

Figure S6. (a-c) IR images showing the state of the programmed film A (microchannel with diameter of $250 \mu \mathrm{m}$ ) at temperature near to the melting point of the gallium. (d-f) IR images showing the shape recovery actuation of the programmed film A under water at $35^{\circ} \mathrm{C}$. 
a

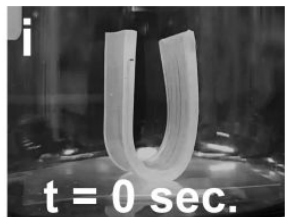

iif

$t=16$ sec.
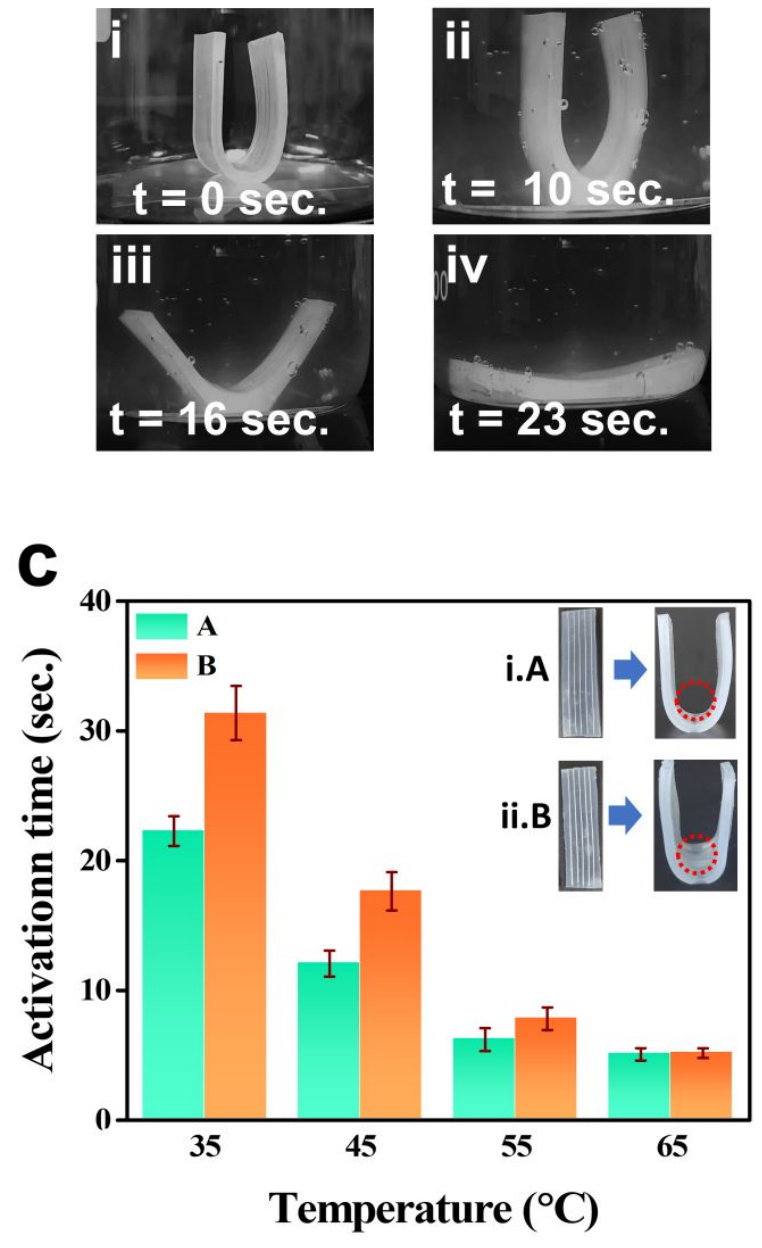
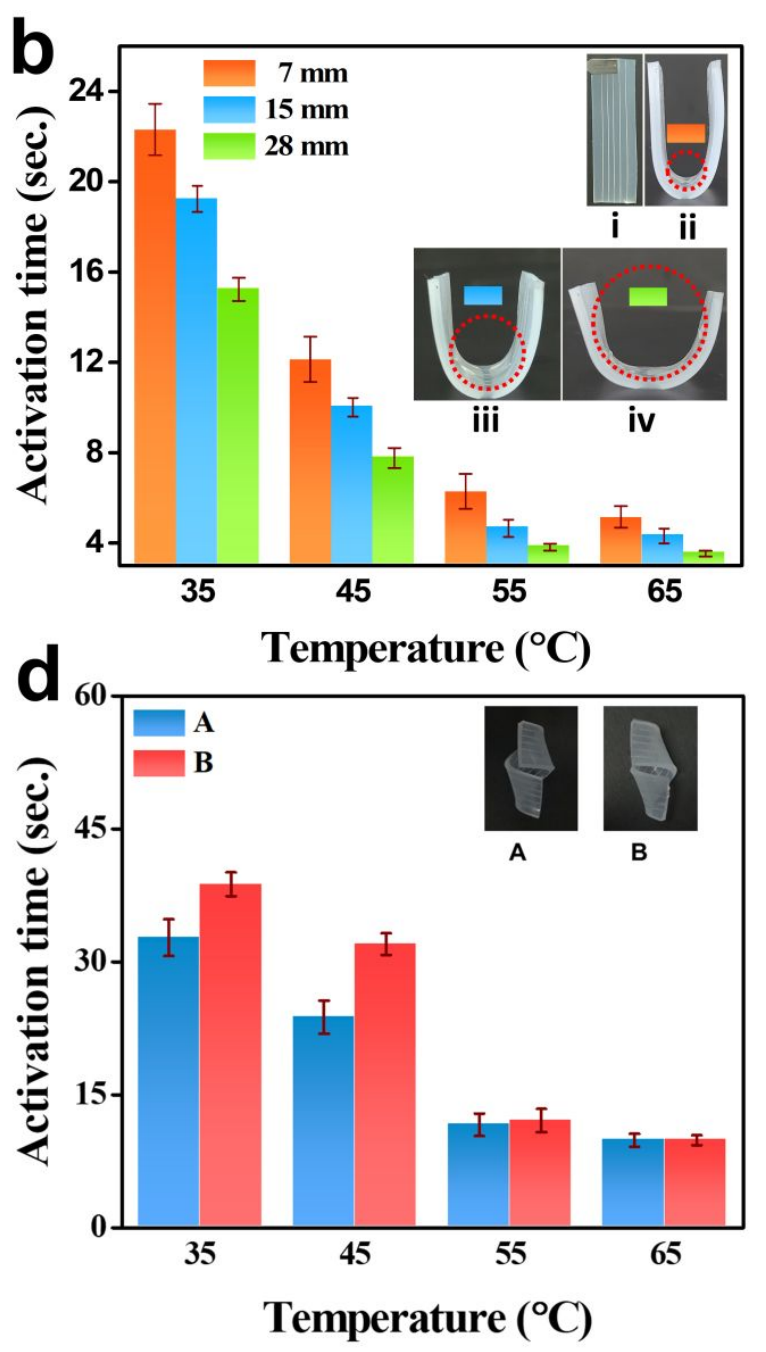

Figure S7. (a) Shape recovery actuation of the film A (microchannel $250 \mu \mathrm{m}$ ) under water at $35^{\circ} \mathrm{C}$.

(b) Activation time of the film (i) A programmed to three different bending diameters of (ii) $7 \mathrm{~mm}$

(iii) $15 \mathrm{~mm}$ (iv) $28 \mathrm{~mm}$ under water at various temperatures. (c) Activation time of the fims (i) A - microchannel $250 \mu \mathrm{m}$ and (ii) B- microchannel $500 \mu \mathrm{m}$ under water at different temperatures.

(d) Activation time of the films A and B programmed to twisted shape (twisting diameter 7mm) 


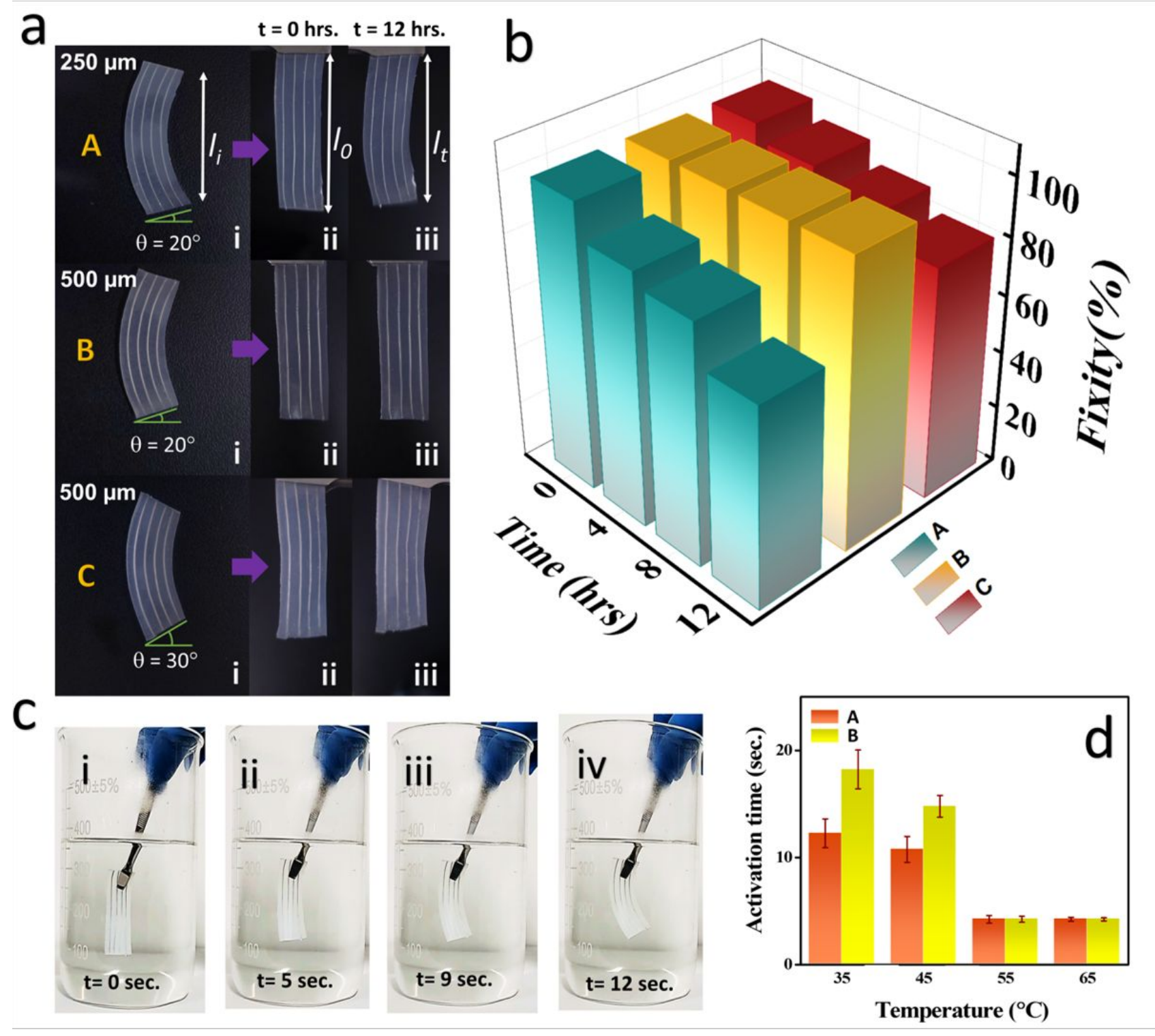

Figure S8. (a) Three bent shaped polymeric films: A (ends cut to an angle $\theta=20^{\circ}$, microchannel with diameter of $250 \mu \mathrm{m}), \mathrm{B}\left(\theta=20^{\circ}\right.$, microchannel with diameter of $\left.500 \mu \mathrm{m}\right)$ and $\mathrm{C}\left(\theta=30^{\circ}\right.$, microchannel with diameter of $500 \mu \mathrm{m}$ ) (each film with concentric gallium arc shaped wires with diameters of curvature $80 \mathrm{~mm}, 75 \mathrm{~mm}, 70 \mathrm{~mm}$ and $65 \mathrm{~mm}$ from left to right) and their linearly programmed states at $\mathrm{t}=0$ and $\mathrm{t}=12 \mathrm{~h}$. It was observed that for $\theta=20^{\circ}$, film A would eventually lose its programmed shape, whereas the programmed shape of the film B would remain intact (100 $\%)$. The ends of the same film were further cut to $\theta=30^{\circ}$ to induce more stress and it was observed 
that it would eventually lose its programmed shape. (b) Fixity values of all the three cases A,B and $\mathrm{C}$, for $12 \mathrm{~h}$ at a time interval of $4 \mathrm{~h}$. Fixity value was caluclated using the equation

$$
\operatorname{Fixity}(\%)=\frac{\varepsilon f(t)}{\varepsilon f(0)} \times 100 \#(1)
$$

where $\varepsilon_{\mathrm{f}(0)}$ is the stress arising at time $\mathrm{t}=0$ and $\varepsilon_{\mathrm{f}(\mathrm{t})}$ is the stress arising at time $\mathrm{t}$. Substituting the values of $\varepsilon_{\mathrm{f}(\mathrm{a})}=\left(1_{\mathrm{a}}-1_{\mathrm{i}}\right) / 1_{\mathrm{i}}\left(\mathrm{l}_{\mathrm{i}}=\right.$ original length, $1_{\mathrm{a}}=$ programmed length at time a). Substituting in equation (1)

$$
\operatorname{Fixity}(\%)=\frac{l_{t}-l_{i}}{l_{0}-l_{i}} \times 100 \#(2)
$$

(c) Shape recovery actuation of the film A in water at $35^{\circ} \mathrm{C}$ (Supporting Video S8). (d) Activation time of the films A and B in water at various temperatures. It is obsserved that at lower temperature, the film A has less shape recovery time than film B, chiefly due to the pesence of malleable solidified gallium wires of lower diameters in film A as compared to that of film B. 


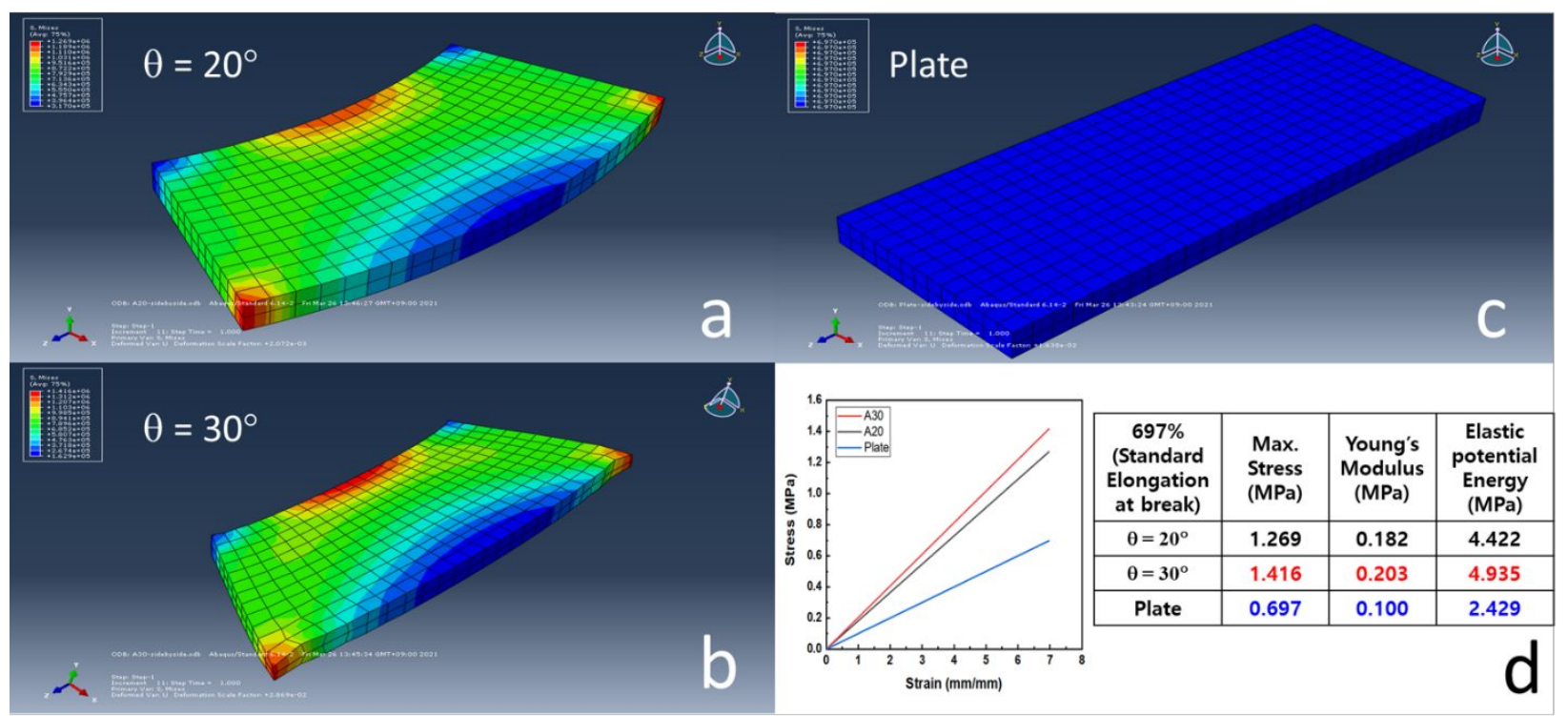

Figure S9. (a-c) FEA analysis showing the stress distribution in the films having end cut angles of (a) $\theta=20^{\circ}$ (b) $\theta=30^{\circ}$ and (c) an unstretched planar film. (d) Mechanical properties of the three systems. 